\section{JSCN T \\ Journal of Sustainable Construction \\ Materials and Technologies}

J Sustain. Construct. Mater. Technol. 3(3) (2018) 249-260
Journal of Sustainable Construction Materials and Technologies

\title{
Rethinking sustainability: A research on starch based bioplastic
}

\author{
Esen Gökçe Özdamar ${ }^{\mathrm{a}, *}$, Murat Ateş ${ }^{\mathrm{b}}$ \\ ${ }^{a}$ Department of Architecture, Faculty of Fine Arts, Design and Architecture, Namık Kemal University, Tekirdağ \\ 59030, Turkey \\ ${ }^{b}$ Department of Chemistry, Faculty of Arts and Sciences, Namik Kemal University, Tekirdağ 59030, Turkey
}

Manuscript Received April 25, 2018; Accepted May 30, 2018

\begin{abstract}
Based on the need to rely on sustainable feedstock, depend less on fossil resources and decrease carbon emissions, biomaterials and bioplastics as substitutes of conventional petroleum based plastics have been the focus of many material scientists, architects and industrial product designers. Therefore, this article is an experimentation on the possibilities of starch based bioplastic production. The focus of the article is to understand the limits of this new material and figure out whether starch based bioplastic material can be used in architecture, both as a facade material and an interior space furnishing.

Based on Steven's bioplastic formula, starch based bioplastic is produced handmade as a surface and cubic specimens with different developed variations in this article. Different starch types, such as potato, corn, wheat and tapioca are tested and mixed with pellets known as local agricultural waste, natural fibers and aggregates. Within the research bioplastic produced from potato starch is formed and molded firstly as a sheet and secondly as a three-dimensional material and tested for vulnerability and durability. The research expands to understanding how organic and inorganic interventions can be made in order to increase the life span of the material, make it durable and resistant to humid and weather conditions. It is observed that tapioca starch gives the finest, smoothest, flexible and strengthful biopolymer among all.

Issues on sustainability, designing and sensing the unpredictable and searching for "new" materials for a greener and sustainable future are the main core of bioplastic production. Regarding the negative carbon footprint and long-term environmental effects of fossil-based plastics through landfill and incineration, the search for such a material brings forth a deeper material experience along with a further collaboration of architects and engineering disciplines. Through this production, we need to figure out deeply the nature of new starch based materials in architecture, which are eco-friendly, cheaper and more strengthful materials compared to conventional synthesized polymers.
\end{abstract}

Keywords: Agricultural waste; biocomposite, construction material, material design, starch based bioplastic.

\section{Introduction}

Today, there is a shift towards circular economy in which energy, resource management and production and consumption patterns have to be reconsidered due to our growing population, excessive consumption and their negative effects on the environment. In the state of a global crisis due to this excessive and irreplaceable

\footnotetext{
* Corresponding author:

E-mail: gokceozdamar@hotmail.com (E. Gökçe Özdamar)
} 
sustainable feedstock use, we need to re-evaluate the production of consumer goods-with a greater focus on food packaging - building and construction materials that consume less energy and continue its lifecycle as minimum waste or different function.

Based on the need to rely on sustainable feedstock, depend less on fossil resources and decrease carbon emissions, biomaterials and bioplastics as substitutes of conventional petroleum based plastics have been the focus of many material scientists, architects and industrial product designers. In response to the environmental problems caused by petroleum-based plastics dumped as waste in the oceans, the development of biocomposite materials such as bioplastics represent a paradigmatic shift, driving the evolution of conventional material practices. There are two major advantages to bio-based plastic products: they save fossil resources by using biomass and provide the keenly sought-after possibility of carbon neutrality. Additionally, bioplastics are often biodegradable [1] (Figure 1).
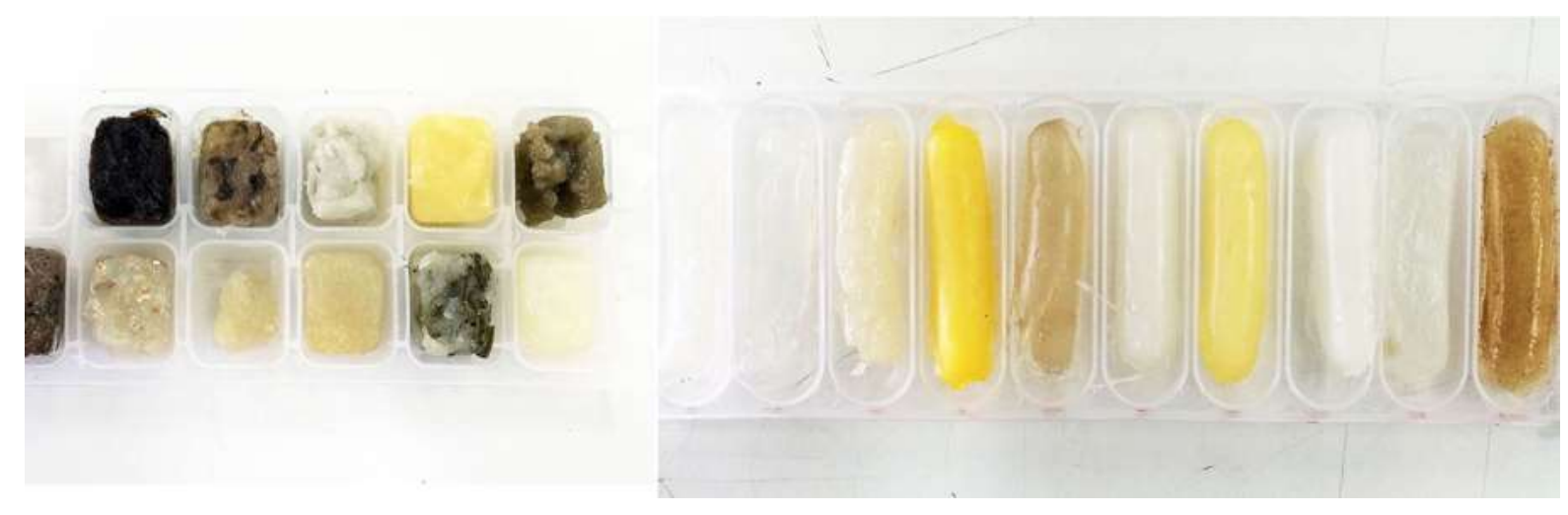

Fig. 1. Simple bioplastic with sand, honey and milk. Photo by the author.

Research on starch based bioplastic in architecture is very new and has a more widespread use mainly in packaging, civil engineering and textile industry. Stevens mentioned biodegradable plastics derived entirely or almost entirely from renewable raw materials [2, vii] and grouped bioplastic into three: bioplastics "made from ready-made polymers in nature, such as starch; plastics processed from polymers produced by microbial fermentation; and plastics processed from resins polymerized from naturally occurring monomers such as lactic acid" [3]. (He formulated starch based bioplastic that was also taken as a reference point in this research for development.

Gupta addressed the use of starch based bioplastic as a packaging material [4]. Pilla evaluated the potential use of bioplastics for its becoming widespread use in the packaging sector, civil engineering, biomedical uses, automotive and general engineering, mainly in consumer goods. There are different of bioplastics derived from starch, as well as polyactic acid (PLA: a starch derived from corn), poly-hydroxybutyrate (PHB), soy based plastics and more [5].

Since bioplastics and specially starch based bioplastics have been researched in engineering disciplines, there is less attempt to discover its uses as a façade element and material in architecture. Some inspirations for experimenting bioplastic and biocomposite materials and products can be given as follows: Marilu Valente, an architecture student designed bioplastic brick and experimented with tensile strength and durability of it (Figure 2). Another project made of bioplastic is Arboskin pavilion in Stuttgart. The pavilion is made up of a bioplastic that contains of materials that are over $90 \%$ renewable, such as starch, cellulose, lignin and biopolymers and is used as a fire exit of a building. The shell is designed with laser-cut modules molded with high temperature and laser-cut surface. To form the structure, the triangular cells that make up Arboskin were linked together with bracing rings and joists [6] (Figure 3). The other projects realized by architects and artists can be given as: "HalfAcre/Half-Life" project by Meredith Miller which is an artistic experimentation of hand-made starch based bioplastic and to observe its decaying and biodegradibility without tests. Other experimental works are Juliette Pepin and Johan Viladrich, two multidisciplinary designers who experiment with small samples of different starch based bioplastic through its variations such as combining bioplastic with different materials such as felt, ash and blood. 

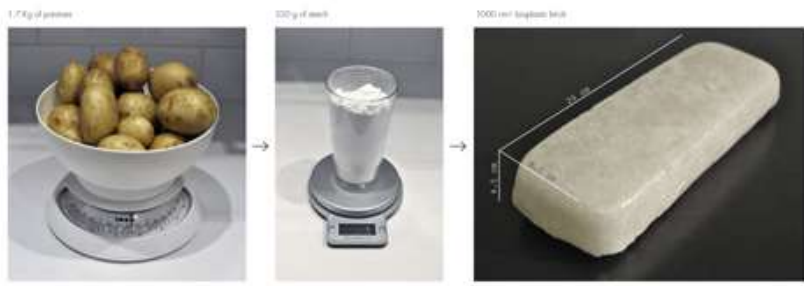

Figure 2. Bioplastic brick by Marilu Valente [7].

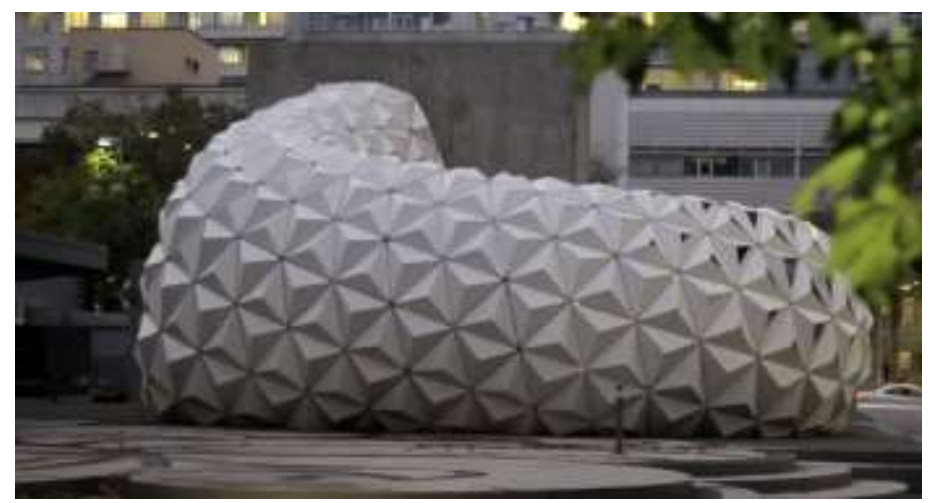

Figure 3. Arboskin pavillion, Stuttgart [8].

Along with these experiments of bioplastic in architecture, further research needs to be done in order to evaluate the performance of starch based bioplastic in architecture. Therefore, deriving from ecological means and the above mentioned examples from an architect or artist approach to bioplastic, this research aims to find out possibilities of starch based bioplastic and biocomposite as a construction material. The focus of this research is to figure out whether starch based bioplastic material can be used in architecture, both as a facade material and an interior space furnishing. The research also aims to understand whether the material can be used alternatively to the cement as a connective material in concrete or perform better when blended with natural and other synthetic fibers. Within the research, vegetable based starch is blended with different additives and biopolymers, natural fibers such as pellet (compressed sunflower, canola and other agricultural waste) and synthetic fibers in order to improve a strength and durable material. The project aims to produce a biocomposite material consisting of organic and inorganic materials that can be used as a surface and furniture. The research expands to understanding how organic and inorganic interventions can be made in order to increase the life span of the material, make it durable and resistant to humid and weather conditions [9].

Within the research, it is also suggested that bioplastic material can also be used alternatively to the cement as a connective material in concrete. Bioplastic produced from potato starch is formed and molded firstly as a sheet and secondly as a three-dimensional material and tested for vulnerability and durability, as well as for understanding material behavior under pressure, humid and different conditions [9].

\section{Starch Based Bioplastic}

Bioplastics are sustainable, largely biodegradable and biocompatible. A simple bioplastic is formed with the following equation: Biopolymer(s) + plasticizer(s)+ other additive $(s)=$ BIOPLASTIC [2, p. 105]. A bioplastic is formed from starch, gelatin/agar, sorbitol, glycerol (glycerin). These ingredients are heated to just below boiling 
$\left(95^{\circ} \mathrm{C}\right)$ in a hot plate and later dried in an oven $[2,166]$ (Figure 4).

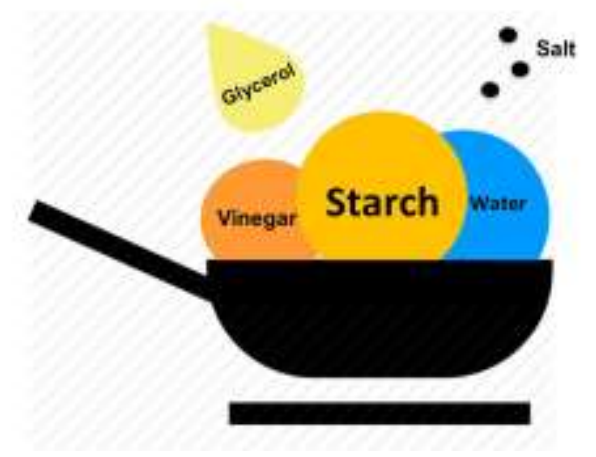

Figure 4. Ingredients of pure bioplastic [10].

Starch is a polysachharide, "generally extracted from the plant by wet milling process" and is used as a starting material for a wide range of green biomaterials. About $75 \%$ of all organic material exists in the form of polysaccharides [4, 189]. Some of different sources of starch are corn, pea, oat, potato/sweet/white, water chestnut, wheat, chestnut, banana, rye, tapioca, etc. [4, 190]. "Starch is used in its raw state or further processed by bacterial fermentation to produce biobased monomers, which are polymerized into bioplastics" [11, 202].

Potato starch is one of the suitable resources for film formation and has a potential use for packaging [12,6]. Food packaging has expecially been one of the most quickly developed markets for starch based bioplastics [2, 149]. When heated, water disrupts starch molecules. Gupta explains this process as: "When dried, the disordered polymer chains become entangled and a neat film is formed". "The granules swell and burst, the semi crystalline structure is lost and the smaller amylose molecules start leaching out of the granule, forming a network that holds water and increasing the mixture's viscosity. This process is called gelatinization. During cooking, the starch becomes a paste and increases further in viscosity. During cooling or prolonged storage of the paste, the semi crystalline structure partially recovers and the starch thickens, expelling water" $[4,191]$.

The process of "heating, mixing and shear stress" breakdown starch granules, "making it a thermoplastics material with interesting tensile properties (modulus and strength) and gas barrier properties" [12, 6]. Vinegar (acetic acid) helps the gelatin and glycerin get together [13].

Today, bioplastics can be found in packaging, food services, agriculture/horticulture, automotive and consumer goods, and this range is only increasing. Bioplastics can be derived from starch, as well as polylactic acid (PLA: a starch derived from corn), poly-hydroxybutyrate (PHB), soy based plastics and more [5, 2].

In the production of starch based bioplastic, Steven's basic formula was referenced, however different proportions and additives were tested to observe and compare the durability, flexibility and resistance to humid of the outcome products. Within this research xxx research group combined architecture and bioplastic and tried to understand how designers gain experience from indeterminate experimentations through materiality. Through questioning a relationship between form, program, process and content hapticity and material experience gained importance. Today, haptic experience can remind us of how to engage with natural and human-made materials, their values and effects on the environment in our world of consumption. This experience is therefore not only a finding out or invention a new material or fetishize innovative materialism, but about rethinking on values, consumption practices and life cycles and decaying and biodegradability of the material. Due the nature to the nature of hand-crafting and cooking skills, and the nature of experiments, handling ambiguity was the core of the experimenting process.

\section{Experiments with Bioplastic and Methodology}

In our experiments, bioplastic is produced from potato starch, firstly as a sheet and secondly as a threedimensional material and tested for vulnerability and durability, as well as for understanding material behavior under pressure, humid and different conditions.

In this study, the inspiration point for such a tendency towards understanding bioplastic materials and producing them was developed by the observation of the dominancy of farming in Tekirdağ, a small waterfront city in the neighborhood of İstanbul. The city's economy depends mainly on farming: The city is covered with sunflowers, canola (95\% of whole production in Turkey) and wheat. The stalks of these plants are evaluated as agricultural waste and cannot be eaten by humans and animals; instead they are compressed and formed into pellet sticks, which are later burnt in special stoves and are effective sources for biodiesel. 
Bioplastic material has two weaknesses. First, bioplastics can be very susceptible to moisture and secondly, it is not a particularly strong material. However, by combining two or three different polymers - such as agar agar, fibers collected from vegetable and fruit waste, arabic gum, resin, coffee grout, or lye (from oak ash)- to form a composite material these weaknesses can be overcome. The use of all additives can also reduce both the cost of the material and $\mathrm{C} 02$ emissions. We also blend edible bioplastic with natural fibers; such as pellet (compressed agglomerates; mainly canola and sunflower waste) and lignin in order to improve moisture susceptibility and strength of bioplastic natural and synthetic fibers.

Bioplastic experiments are classified under 4 groups: Pure bioplastic, bioplastic as a matrix for aggregates, bioplastic with different fibers, and different fibers to improve properties of bioplastic. These blends were compared to each other.

\subsection{Pure bioplastic}

Pure bioplastic was gained with $6 \times 6 \times 6$ and $15 \times 22 \times 0,8 \mathrm{~cm}$ steel and wooden molds. It was observed that pure bioplastic specimens are not resistant to humid and compression and cracked. Potato, corn, wheat and tapioca starch were used and it was observed that tapioca starch works as the best type of biopolymer in the formation of bioplastic and secondly the potato starch, thirdly wheat starch. The figures show pure three-dimensional specimens (Figure 5).
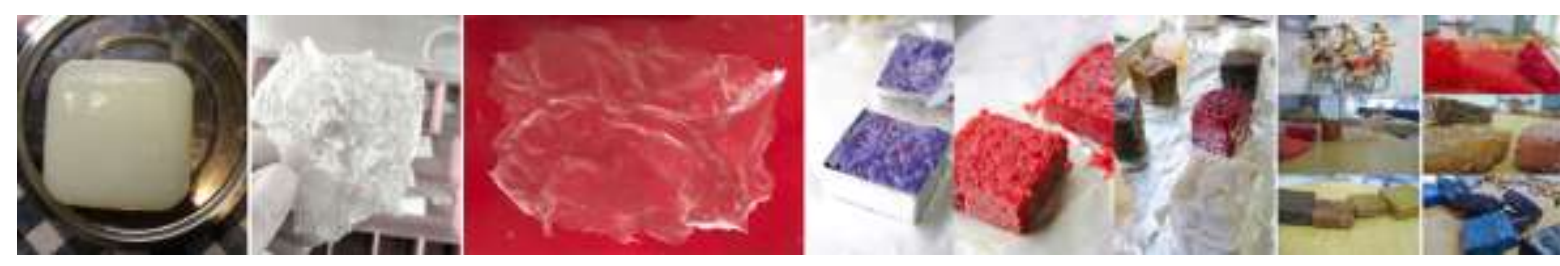

Figure 5. Pure potato and corn starch bioplastic, potato starch in sheet form and corn starch colored modules from the exhibition, realized in a workshop during Tirana Architecture Weeks by students of Polis University in Tirana. Photos by the author and Julia Janku.

\subsection{Bioplastic as a matrix for aggregates}

Pure bioplastic was mixed with 0-4 mm. aggregates in order to improve the properties of strength. These specimens were molded with $15 \times 15 \times 15 \mathrm{~cm}$ steel concrete molds. The results were observed as sponge-like, lightweight material with some cracks on the surface due to open-air drying (Figure 6).
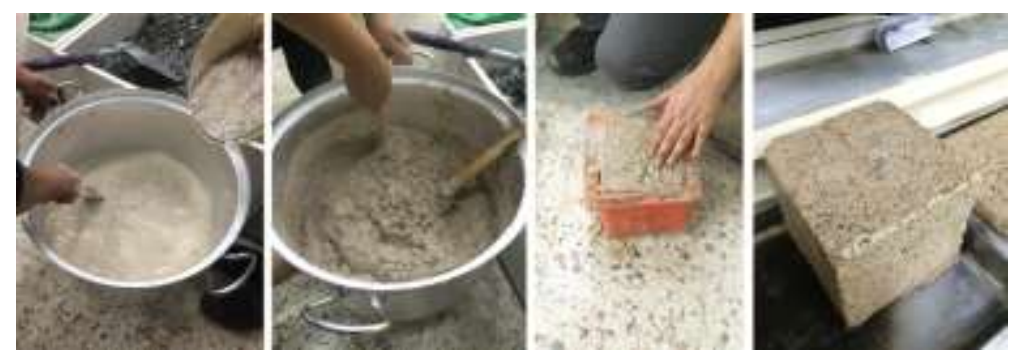

Figure 6. 50\% Bioplastic, $50 \%$ Mixture: (Aggregate:0/4 mm), Steel mold, Dimension: 15 x 15 x 15 cm. Photo by the author.

\subsection{Bioplastic as a matrix for different fibres}

Due to the difficulties in making a homogeneous mixture in $15 \mathrm{~cm}$ cubic specimens, $5 \mathrm{~cm}$ and $6 \mathrm{~cm}$ cubic specimens were gained. Agar agar, synthetic polyolefin fibers and pellets were added. Additionally, beewax, cotton, gauze band, agar agar, oak mesh and lye, gum tragacanth, arabic gum, pine resin and pellet were added. It was observed that these additions strengthen bioplastic (Figure 7). 


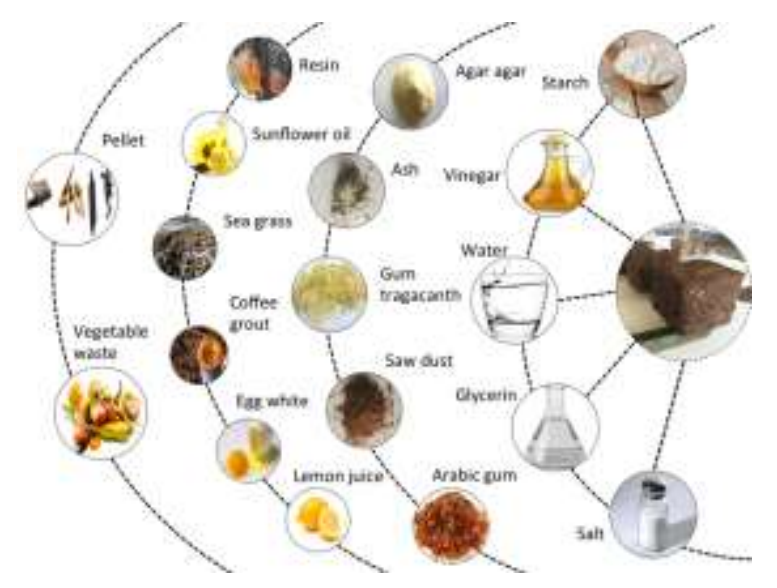

Figure 7. Used additives in order to improve mechanical properties of bioplastic; biopolymers and plasticizers for bioplastic.

Agar agar (E406) is a biopolymer and a polysaccharide derived from red seaweed and is used in food industry. In the experiments agar was used in powder form $[2,166]$. Bioplastic mixture was poured in liquid jelly form which resulted as cracks on the surface. Instead solid mixture displayed much better results. It was observed that agar agar acts as a strong biopolymer for starch based bioplastics, as well as dried skin of various fruit and vegetables such as pumpkin seed, fig and corn leaf.

When the blend is poured on a natural rubber or EVA surface rather than steel, glass or wood surface, less crack or deformation on the surface was formed (Figures 8, 9, 10).
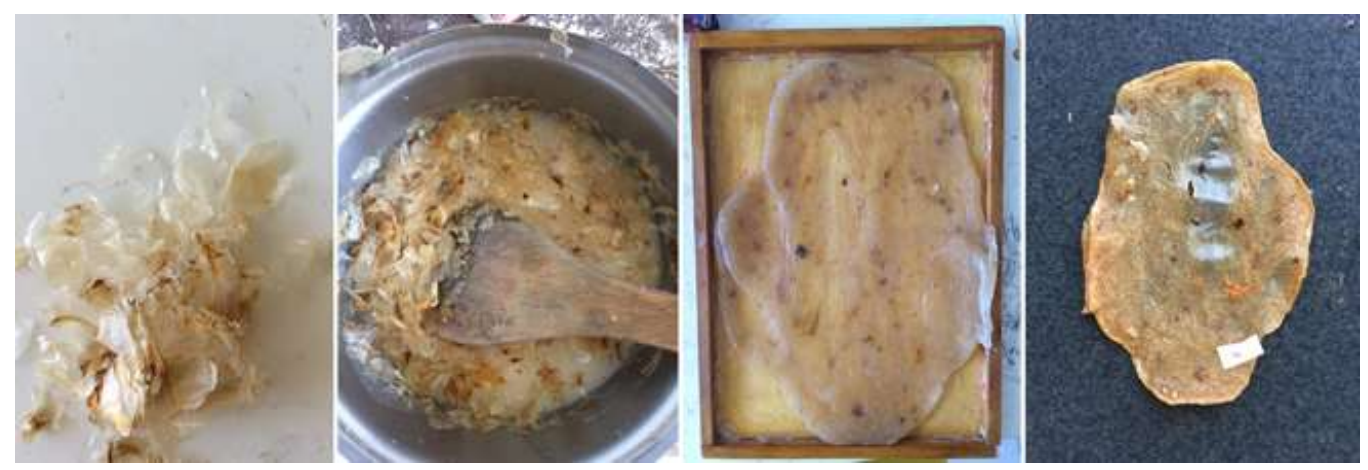

Figure 8. Dried skin of pumpkin seed as a biopolymer with agar agar potato starch bioplastic. Photo by the author.

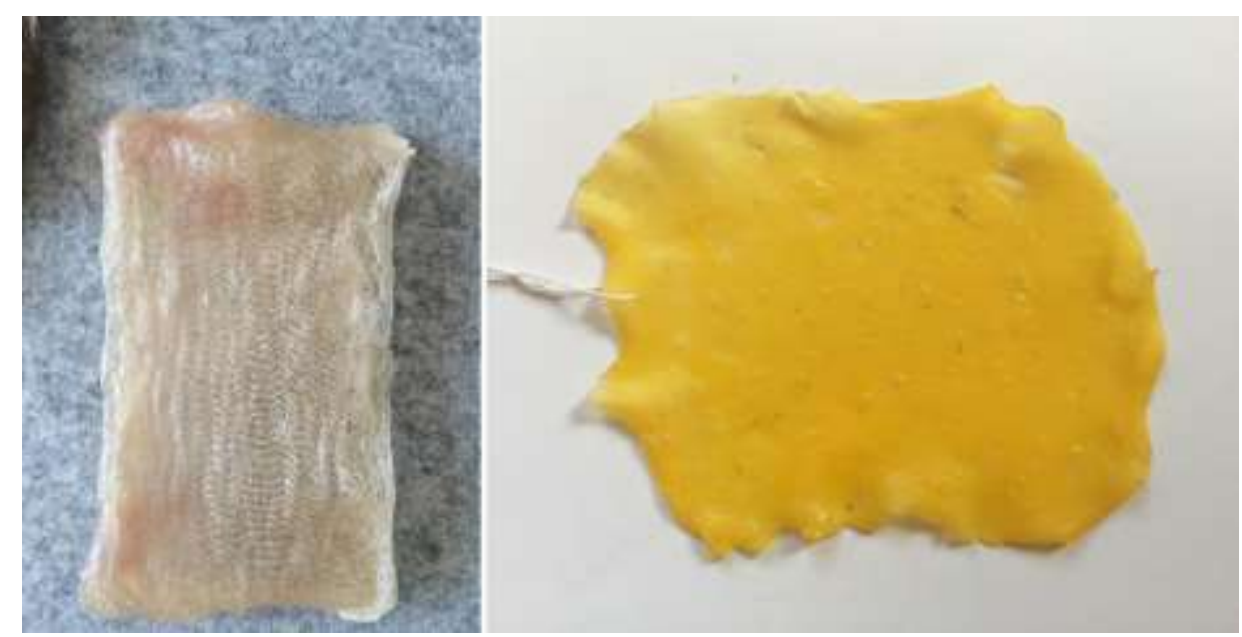

Figure 9. Tapioca starch with agar agar bioplastic. Photo by the author. 


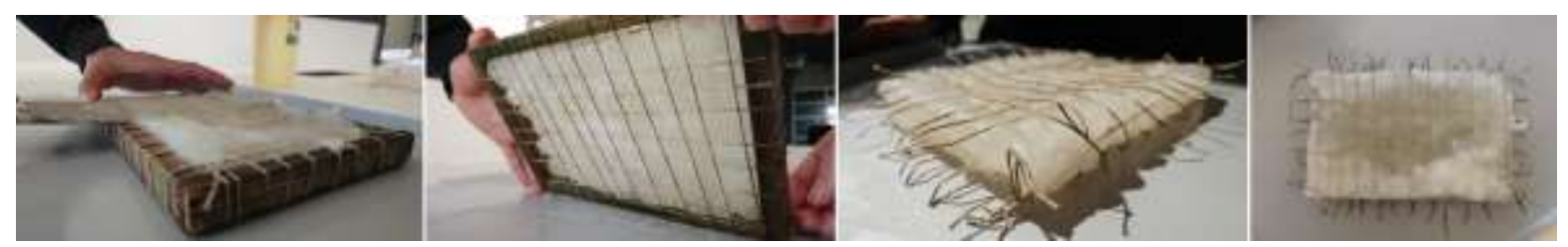

Figure 10. Bioplastic with linen grid, Dimension: $15 \times 22 \times 0,8 \mathrm{~cm}$. Photo by the author.

\subsubsection{Pellet as Agricultural Waste for Bioplastic}

Pellets are compressed agglomerates used for making biodiesel such as canola or sunflower waste. They help save fuel resources. In our experiments, canola pellets were used in $6 \times 6 \times 6 \mathrm{~cm}$ and $15 \times 22 \times 0,8 \mathrm{~cm}$ specimens. It was observed that pellet use increases the endurance of the specimen, making it strengthful. Sea grass (Posidonia oceanica) has been used by Neptutherm firm as an insulation material. It was observed that the use of small particles of this seaplant provide sufficient hardness and endurance for bioplastic (Figures 11, 12, 13).
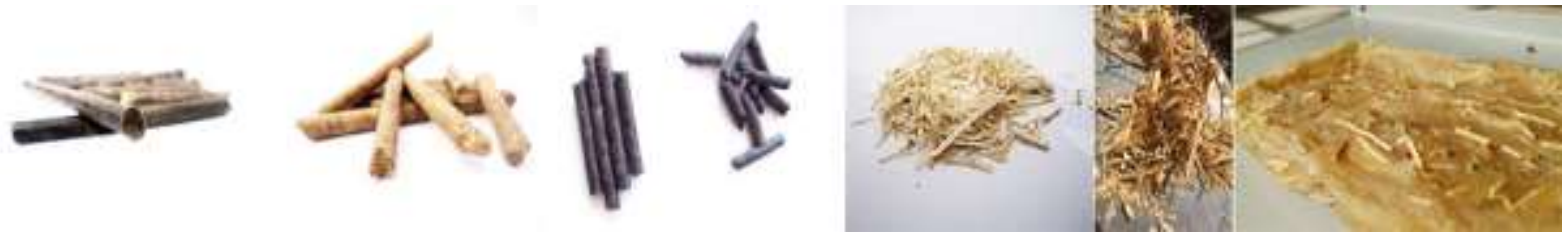

Figure 11. Pellets and natural fibres used in experiments, 1. Canola stalk pellet, 2. molasses pellet, 3. canola and coal dust pellet, 4. molasses and ash pellet [From left to right] and different natural fibers, Dimension: $15 \times 22 \times 0,8$ $\mathrm{cm}$. Photo by the author.
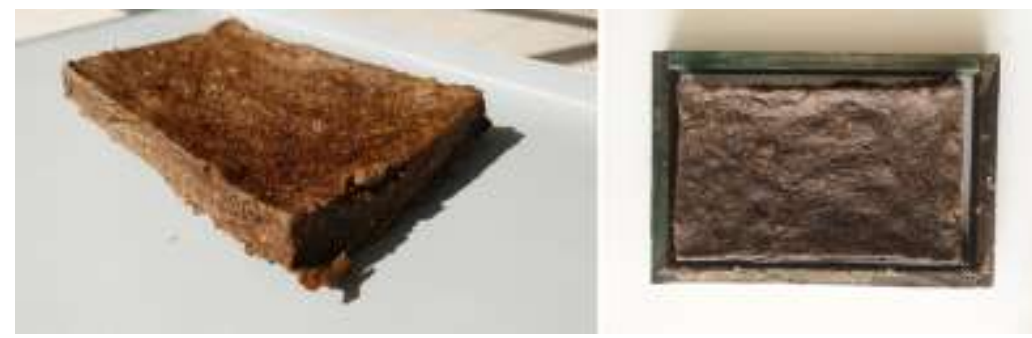

Figure 12. Canola pellet with bee wax, Dimension: $15 \times 22 \times 0,8 \mathrm{~cm}$. Photo by the author.

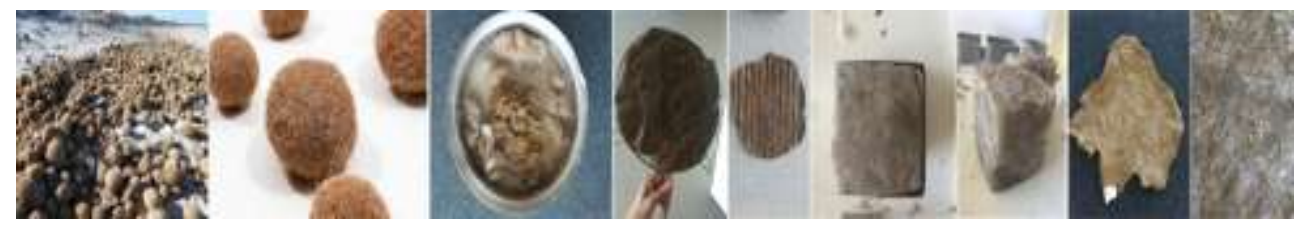

Figure 13. Seagrass (Posidonia oceanica) collected from shores of Albania, [14, 15]. Seagrass and canola pellet potato starch bioplastic and pure seagrass potato starch bioplastic, Dimension $5 \times 5 \times 5 \mathrm{~cm}$ and potato starch bioplastic with seagrass. Photo by the author.

\subsection{Addition of different fibers to bioplastic which are matrix to aggregates}

In this group of experiments, marble powder was added to $15 \times 15 \times 0,3 \mathrm{~cm}$ specimens and was found out that the materials were hard. Regarding all these groups, modules of $6 \times 6 \times 6 \mathrm{~cm}$ with potato starch and agar agar were combined in order to make a lateral wall. The mortar was used by a mixture of lime with gypsum and mixed aggregate of $0 / 1$ and $0 / 4 \mathrm{~mm}$. This mortar was also dried in the oven (Figures 14, 15, 16). 

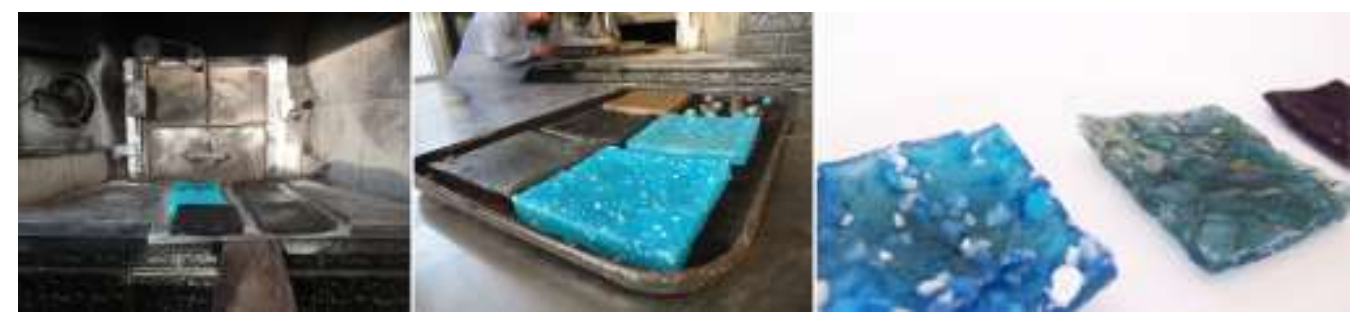

Figure 14. Marble aggregate and synthetic fiber bioplastic with methylene for color, Dimension: $15 \times 15 \times 0,3$ and $5 \times 5 \times 0,3 \mathrm{~cm}$. Photo by the author.
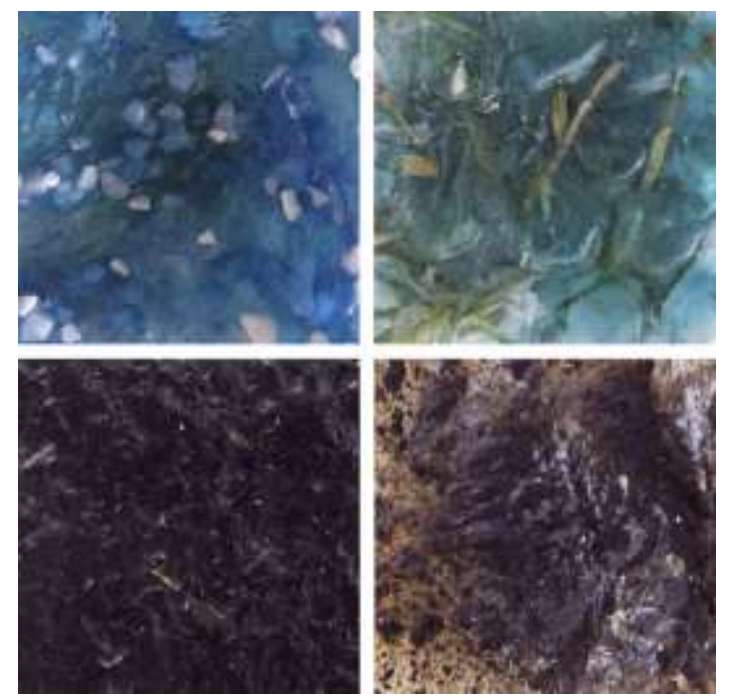

Figure 15. Specimens $5 \times 5 \times 0,3 \mathrm{~cm}$ : 1 . Bioplastic with marble powder, methylene, 2. Bioplastic with wood fiber, methylene, 3. Bioplastic with pressed canola stalk, coal dust pellet, lye, 4. Bioplastic with pressed coffee, lye (From left to right in rows). Photo by the author.

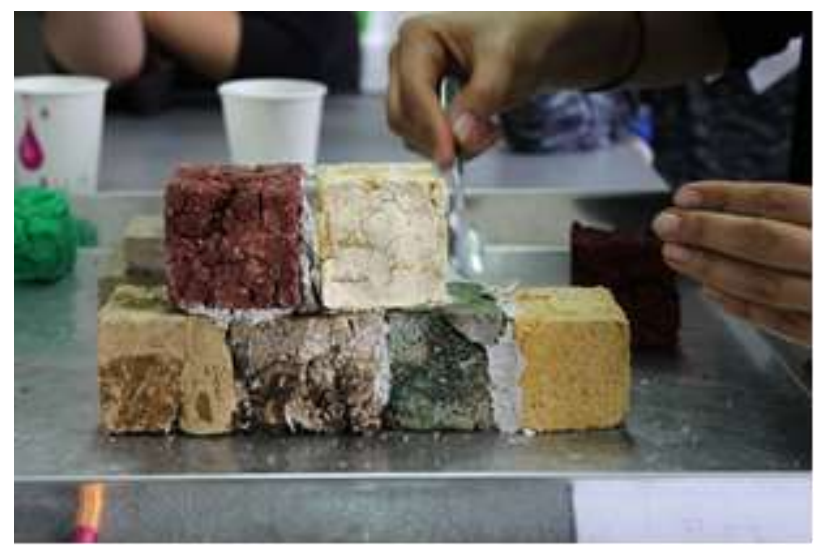

Figure 16. 6x6x6cm cubic modular wall. Photo by the author.

\section{Chemistry of Bioplastic}

Starch has a heterogeneous, semi-crystalline granular structure and the degree of ordered structure are important effect in foods and bioplastic formation [16]. Starch is one of the biodegradable polymers which can replace synthetic polymers in situations requiring short-term solutions, such as food packaging and disposable cutlery. Major starch resources include potato, corn, rice, wheat gluten and soy. In these starch is stored in the form of semi-crystalline granules composed of two glucose polymers, amylose and amylopectin [12, 5-6]. In light of recent research carried out at Stanford University, it seems the next generation of bioplastics will be 
derived from carbon dioxide.

Other recent research has studied a reinforcement for wheat gluten based bioplastics produced from rubber and sawdust. The experiment, carried out by Bootklad et al., evaluated the morphology, water absorption, mechanical, thermal and biodegradation properties of the wheat gluten and found good dispersion and uniform embedding in the material [17]. In this article, a few characterization techniques were evaluated for rubber wood sawdust (RWS), such as morphology, water absorption, mechanical, thermal and biodegradation properties of the wheat gluten. A good dispersion and uniform embedding of the EWS was observed within the wheat gluten matrix.

With high moisture and intermediate moisture materials, such as gels, cakes, and breads, starch bioplastics include only a limited amount of water (less than $20 \% \mathrm{w} / \mathrm{w}$ ) both during processing and after processing [18]. Starch structure can be characterized by differential scanning calorimetry, ${ }^{13} \mathrm{C}$-nuclear magnetic resonance, $\mathrm{X}$ ray diffraction and Fourier-transform infrared spectroscopy (FTIR). Native potato starch was derivatized using different agents to reduce its hydrophobicity. If we add nanocellulose into the starch material, the mechanical properties of biopolymers as well as to maintain their inherent biodegradable capability will improve [19].

This contribution demonstrates a new approach to form bioplastics with both improved moisture and heat resistance and high mechanical properties. Further research on morphology and structure is essential to understand the structure-property relationship of polymer composites [20]. The development of biodegradable materials to be used by the plastic industry has been taken increasing interest over the last few years. GonzálezGutierrez et al [21] have studied the different blends of albumen protein and starch (potato and corn), which are used as raw materials for bioplastics exhibiting high transparency and suitable mechanical properties.

However, long-term issues such as recycling bioplastic from petroleum-based plastics, the cost, and reliability on the soil remain controversial issues. Recycling feedstock such as pellet and natural fibres that cannot be eaten by humans and animals are good resources in for bioplastic in terms of waste management.

\section{Biodegradability}

'In accordance with ASTM D 6400, biodegradable plastics are only those whose degra- dation occurs as a result of natural action of microorganisms, such as bacteria, fungi, and algae, in a limited period of time and in absence of ecotoxic effects' [22, 1950]. Indeed, with regard to recycling, nowadays processes for selecting and recycling bioplastics are not yet developed, despite what happens for conventional plastics [22, 1951].

'Additives are often present in the bioplastics, mainly to improve the mechanical properties of the obtained material. This choice may not only cause a reduction in the biodegradability of plastics and other serious ecotoxic effects, but it might even determine the non-compostability of the bioplastic, making in fact, a vain every advantage achievable through the use of a bioplastic' [22, 1951].

In the experiments, some of the specimens were moulded and beewax mixed specimens shows signs of melting and creating humid internally after a duration of one year. According to the results of experiments in 4 groups, the evaluations are made for specimens under $15 \mathrm{~cm}$ cubic and sheet specimens, since greater size demanded mechanical devices in order to mix the mixture rather than handmade. It is observed that soft fiber additions increase in bioplastic material strength. Agar agar (biopolymer), seagrass, gum tragacanth, and pellet addition effect bioplastic in terms of durability. Cube bioplastic specimen is tested under the one directional axial force for compressive strength. Cube specimen consists of 50\% bioplastic and 50\% aggregate. The aggregate used in the bioplastic cube specimen is $0-4 \mathrm{~mm}$ in size. After the compressive strength test was performed after 28 days without cooking, molding and open air drying. Compressive strength of bioplastic cube specimens, 2.8MPa, 3.2MPa and 3.05MPa. Average strength of material is 3.02 $\mathrm{MPa}[10]$.

The results obtained from our recent experiments show that bioplastics can be used as construction materials in the form of three-dimensional elements under available conditions. However, durability, strength conditions, moist susceptibility and life cycle of the material need to be evaluated within further research [10].

\section{Bioplastic Materials in Architecture}

Recent advances in the mechanistic development of bioplastic materials and the computer-aided design approach and robotics have improved the ability to construct more sustainable architectural systems [23]. In academic literature, there are many bioplastic material usage in numerous biomedical and nanotechnologic applications, such as tissue engineering "smart nanodevices" drug delivery, and protein purification [24]. Molecular biologists are exploring bioplastic are exploring biosynthetic compounds to non-natural proteins with controlled material properties. Scientists have investigated the new developments from molecular biology to polymer chemistry [25].

Besides its recently growing area in chemistry, how will the future of bioplastic influence architects to use this not much practiced ambiguous, yet promising material? To answer this question, we need to evaluate how 
architects and designers gain material experience. It is firstly through confrontation with the material, which means observing designed surface. Secondly, gaining observing is through experimenting with it like an alchemist. The second feature seems to arise. In this age of sustainability, recycle and reuse have brought along ambiguous and indeterminate processes that have led to experimenting with different materials and composite elements.

Currently, the production and the process of biodegrading bioplastic materials is more expensive than conventional plastics. This is due to the complex biological processes in measuring biodegradability and material properties such as tensile strength, indentation hardness, elongation, loss of volatile components, barrier to oxygen and moisture, oil resistance, ease of marring, fold resilience, tear resistance according to (ASTM) American Society for Testing and Materials (ASTM) [2, 180]. Despite this hard, yet surmountable issue, we need to radically change our consumption practices, recycle and minimize the amount of conventional plastic production. Regarding sustainability, lifecycle, life span of materials, the feedstock used for materials, the use and meaning value of materials, we need to understand biological systems in relation to architecture and design.

The future of bioplastics seems to face two confrontations. Firstly, there is a growing trend in the biobased material production. Between new materialisms and consumption innovate a new material, can largely become part of a material fetishism. Secondly, it can create a paradigm shift by replacing conventional plastics with its reduced costs and large availability, as packaging and building construction. Thirdly, bioplastics can find a place in niche-market as Stevens mentions [2, 159].

In all cases, material experience of such vegetable based material production can help minimizing global environmental problems caused by the increasing use of fossil resources in our age of consumption. Regarding our over engineered plastics in order to make strength and durable materials for everyday use, environmental recycling consideration has been a less minor issue. Material experience of such vegetable based material production can help minimizing global environmental problems caused by the increasing use of fossil resources in our age of consumption.

Bioplastics can derive the future of architecture as green composites, which provide a "sustainable alternative with $100 \%$ biodegradability" conventional methods of production, reducing "the carbon foot print on the environment' [5, xxi]. The current experiments for testing the biodegradability of the material is limited due to its expensive costs as well as the complex processes to the biological and complex processes [2, 180]. Additionally, they can enable reducing "food waste" which is a "major source of CO2 emissions" and "keeping renewable carbon in the "loop" "when recycled [26].

\section{Conclusion}

The research aimed at raising curiosity in sustainable and experimental biobased material design by enabling an ambiguous and practice based design process from the perspective of different disciplines. Issues on sustainability, designing and sensing the unpredictable and searching for "new" materials for a greener and sustainable future were the main core of bioplastic production. As a result, a starch based bioplastic materials are constituted from vinegar, starch, glycerol, salt and water for possible use as an emerging innovative material in architecture.

Regarding the negative carbon footprint and long-term environmental effects of fossil-based plastics through landfill and incineration, the search for such a material brings forth a deeper material experience along with a further collaboration of architects and engineering disciplines. Through this production, we need to figure out deeply the nature of new starch based materials in architecture, which are eco-friendly, cheaper and more strengthful materials compared to conventional synthesized polymers.

\section{Acknowledgements}

Bioplarch is a scientific research project originally entitled as "Bioplastic: An Experimental Biomorphology in Architecture" and conducted by Esen Gökce Ozdamar, (With researchers Instructor Ahmet Bal (Construction Engineering), Şermin Şentürk (Architecture student) and Professor, PhD Murat Ates (Chemistry) at Namık Kemal University between 31.03.2016-14.11.2017. This work was supported by Research Fund of the Namik Kemal University under Grant NKUBAP.08.GA.16.050.

The project was also selected in a competition held by Future Architecture Platform, the first pan-European platform of architecture museums, festivals and producers, supported by European Union Creative Europe Program and the project was awarded with a series of conferences and workshops held in Europe in 2016 and 2017. 


\section{References}

1. European Bioplastics (a), 2016. http://en.european-bioplastics.org/bioplastics/ (Date of Consult: 10.01.2016).

2. Stevens, E. S. (2002). Green Plastics: An Introduction to the New Science of Biodegradable Plastics, Princeton University Press. Princeton and Oxford.

3. Frech, C. B. (2002). Green Plastics: An Introduction to the New Science of Biodegradable Plastics, Book \& Media Reviews, Journal of Chemical Education, Volume: 79, Issue: 9.

4. Gupta, K. M. (2011). Starch Based Composites for Packaging Applications. Handbook of Bioplastics and Biocomposites Engineering Applications, S. Pilla, ed., Scrivener Pub., Hoboken, NJ, Wiley, Salem, Mass., 189-262.

5. Pilla, S. ed. (2011). Handbook of Bioplastics and Biocomposites Engineering Applications, Scrivener Pub., Hoboken, NJ, Wiley; Salem, Mass.

6. Arboskin, (2016). https://www.trendhunter.com/trends/bioplastic (Date of Consult: 12.12.2017).

7. Bioplastic Morphologies, (2016). http://www.merged-vertices.com/portfolio/bioplastic-morphologies-2/ (Date of Consult: 10.01.2016).

8. Arboskin pavillion, Stuttgart, (2016). http://designplaygrounds.com/deviants/arboskin-bioplasticfacade-research-itke/ (Date of Consult: 10.01.2016).

9. Özdamar, E. G., and Bal, A. (2016). A "Material Experience" in the Age of Consumption: Bioplarch. Future Architecture Platform. http://futurearchitectureplatform.org/news/21/a-material-experience-inthe-age-of-consumption- bioplarch/ (Date of Consult: 10.11.2017).

10. Özdamar, E. G., and Bal, A. (2017). Investigating Starch Based Bioplastic as A Construction Material. ICBEST, International Conference On Building Envelope Systems and Technologies, Interdisciplinary Perspectives for Future Building Envelopes (Tavil, A., Çelik, O.C. eds. Istanbul, Istanbul Technical University, 564-579.

11. Karana, E., et al. (2014). Materials Experience: fundamentals of materials and design, ButterworthHeinemann, Oxford.

12. Muneer, F. (2014). Bioplastics from natural polymers. Introductory paper at the Faculty of Landscape Architecture, Horticulture and Crop Production Sciences 2014:4, Swedish University of Agricultural Sciences, Alnarp. https://pub.epsilon.slu.se/11915/1/muneer_f_20150220.pdf (Date of Consult: 10.12.2017).

13. Green plastics, (2011). http://green-plastics.net/posts/69/qaa-why-water-and-vinegar/(Date of Consult: 20.10.2016).

14. http://www.neptutherm.com/phpwcms/index.php?home (Date of Consult: 10.11.2016).

15. https://materia.nl/material/neptutherm/ (Date of Consult: 10.11.2016).

16. Warren, F.J., et al. (2016). Infrared spectroscopy as a tool to characterize starch ordered structure-a joint FTIR-ATR, NMR, XRD and DSC study, Carbohydrate Polymers, Volume: 139, 35-42. doi: 10.1016/j.carbpol.2015.11.066.

17. Bootklad, M., et al. (2016). Novel biocomposites based on wheat gluten and rubber wood sawdust, J. Appl. Polym. Sci. Volume: 133, Issue:30, 43705, doi: 10.1002/app.43705.

18. van Soest, J.J.G., and Knooren, N. (1997). "Influence of glycerol and water content on the structure and properties of extruded starch plastic sheets during aging, J. Appl. Polym. Sci., Volume: 64, Issue: 7, 1411-1422. doi: 10.1002/(SICI) 1097-4628(19970516)64:7<1411::AID-APP21>3.0.CO;2-Y.

19. Morán, J.I., et al. (2013). Bio-nanocomposites based on derivatized potato starch and cellulose, preparation, and characterization, J. Mater. Sci., Volume: 48, Issue:20, 7196-7203. doi: 10.1007/s10853-013-7536-x.

20. García, N.L., et al. (2009). Physico-mechanical properties of biodegradable starch nanocomposites, Macromol. Mater. Eng. Volume: 294, Isuue: 3, 169-177. doi: 10.1002/mame.200800271.

21. González-Gutiérrez, et al. (2011). Effect of processing on the viscoelastic, tensile and optical properties of albumen/starch-based bioplastics, Carbohydrate Polymers, Volume: 84, Issue: 1, 308-315. doi: 
10.1016/j.carbpol.2010.11.040.

22. Gironi, F., and Piemonte, V. (2011). Bioplastics and Petroleum-based Plastics: Strengths and Weaknesses, Energy Sources, Part A: Recovery, Utilization and Environmental Effects, Volume: 33, Issue: $21,1949-1959$. doi: 10.1080/15567030903436830.

23. Nawroth, J.C., et al. (2012). A tissue-engineered jellyfish with biomimetic propulsion, Nature Biotechnology, Volume: 30, Issue: 8, 792-797. doi:10.1038/nbt.2269.

24. Girotti, A., et al. (2011). Elastin-like recombinamers: Biosynthetic strategies and biotechnological applications, Biotechnology Journal, Volume: 6, Issue: 10, 1174-1186. doi: 10.1002/biot.201100116.

25. Barron, A. E., and Zuckermann, R.N. (1999). Bioinspired polymeric materials: in between proteins and plastics, Current Opinion in Chemical Biology Volume: 3, Issue: 6, 681-687, doi: 10.1016/S13675931(99)00026-5.

26. European Bioplastics (b), (2015). http://en.european-bioplastics.org/wpcontent/uploads/2015/publications/EUBP_Considerations_Circular_Economy_Proposal_2015.pdf (Date of Consult: 10.01.2016). 